(C) 2000 IEEE. Personal use of this material is permitted. Permission from IEEE must be obtained for all other uses, in any current or future media, including reprinting/republishing this material for advertising or promotional purposes, creating new collective works, for resale or redistribution to servers or lists, or reuse of any copyrighted component of this work in other works. 


\title{
Scintillator Identification and Performance Characteristics of LSO and GSO PSPMT Detector Modules Combined through Common X and Y Resistive Dividers
}

\author{
J. Seidel, J. J. Vaquero, Senior Member, IEEE, F. Barbosa ${ }^{\#}$, Member, IEEE, \\ I. J. Lee, C. Cuevas", Member, IEEE, and M. V. Green \\ National Institutes of Health, Bethesda, Maryland 20892 \\ \#Thomas Jefferson National Accelerator Facility, Newport News, Virginia 23606
}

\begin{abstract}
Combining signal channels from detector arrays can reduce complexity and minimize cost but, potentially, at the expense of other performance parameters. We evaluated a method that reduces the number of signals by combining the anode outputs of three position-sensitive photomultiplier tubes (PSPMTs) through a common $\mathrm{X}$ resistive charge divider and three individual $\mathrm{Y}$ resistive charge dividers. Field flood images at $511 \mathrm{keV}$ of two LSO modules combined with a single GSO module were compared to images obtained when the modules were illuminated separately. At moderate count rates only a small reduction in position detection accuracy was observed in the combined tubes. Event mis-positioning was minimal for total count rates $<300,000 \mathrm{cps}$. At higher rates, pulse pileup degraded accuracy. Delayed charge integration, a method for identifying scintillators by differences in their light decay times, allowed the LSO and GSO arrays to be distinguished from one another and also reduced the effect of pulse pileup. Thus, combining PSPMTs anodes through common $X$ and common $\mathrm{Y}$ resistive dividers may be useful in reducing signal number from PSPMT detector modules while maintaining good event localization and scintillator identification accuracy at reasonable event rates.
\end{abstract}

\section{INTRODUCTION}

A common method of reducing the number of signals from PSPMTs is to combine all $\mathrm{X}$ anode signals from a tube through a resistive divider and all $\mathrm{Y}$ anode signals through another resistive divider [1-3]. The position of a scintillation event within a module is then calculated from the four signal outputs from these two chains rather than from the full number of anode signals. However, even with this signal compression scheme the total number of signal channels can still be large if the number of tubes is large.

Since PSPMT anodes can be considered ideal current sources, a further compression in signal number might be possible by combining all of the $\mathrm{X}$ anode signal outputs from multiple PSPMTs through the same resistive charge divider and all $\mathrm{Y}$ anode signal outputs through a second resistive charge divider. This scheme reduces the number of signal channels to four (two for the $\mathrm{X}$ position, two for the $\mathrm{Y}$ ) regardless of the number of tubes that are combined.

The disadvantage of this approach is that the count rate independence of each tube is lost and the event rate seen by the combination is the sum of event rates in each tube. Thus, pulse pileup at high rates may adversely affect event positioning. Accordingly, we measured position detection accuracy at moderate count rates to assess the effect of combining tubes by threes and then, when combined, characterized event positioning as count rate increased.

In addition, we sought to determine whether "delayed charge integration" (DCI, [4]), a method of identifying the scintillator-of-interaction through differences in light decay time, might be compromised by the increase in time constant associated with resistive charge division. DCI depends on pulse shape and could fail to distinguish between two fast scintillators (LSO and GSO) that differ only by $20 \mathrm{~ns}$ in decay time. Pulse pileup at high rates also leads to pulse shape degradation so we also investigated the dependence of the DCI method on count rate.

\section{MATERIALS AND METHODS}

Three detector modules were fabricated. Each was comprised of a $9 \times 9$ arrays of $2 \mathrm{~mm} \times 2 \mathrm{~mm} \times 10 \mathrm{~mm}$ crystals (2.2 mm pitch) optically coupled to Hamamatsu R5900-C8 position sensitive photomultiplier tubes. Each crystal was double-wrapped with Teflon tape on all but the exit end. The crystal arrays were optically coupled to the PSPMTs with silicon grease (V-788, $\mathrm{n}=1.47$, Visilox Systems, Troy, New York) and enclosed in light-tight boxes.

Two of the modules were comprised of LSO crystals (CTI, Knoxville, TN, light decay time $=40 \mathrm{~ns}$ ) and one of GSO crystals (Hitachi, Japan, light decay time $=60 \mathrm{~ns}$ ). The LSO crystals were mechanically ground and polished (3D Precision Optics, Ravenna, $\mathrm{OH}$ ) on all but the entrance end which was diffusely ground. The GSO crystals were chemically etched on all sides (Hitachi).

In order to establish reference performance values for the modules before being combined, experiments were carried out with the four $\mathrm{X}$-anode and four $\mathrm{Y}$-anode outputs of each tube connected to their own individual $\mathrm{X}$ and $\mathrm{Y}$ resistive charge dividers.

The anodes of the three modules were then combined as shown in Figure 1. In this arrangement, all of the X-anode signals are combined through a single common charge divider, compressing the number of $\mathrm{X}$ signals from a maximum of 12 down to two. The two signals (XA, XB) emerging from this divider allow the $\mathrm{X}$-position of an event occurring in any of the three modules to be determined. The number of $Y$ signals is also reduced from 12 to two (YA, YB). In this case, however, three identical resistive charge dividers are used to combine the $\mathrm{Y}$-anode signals rather than just one. 


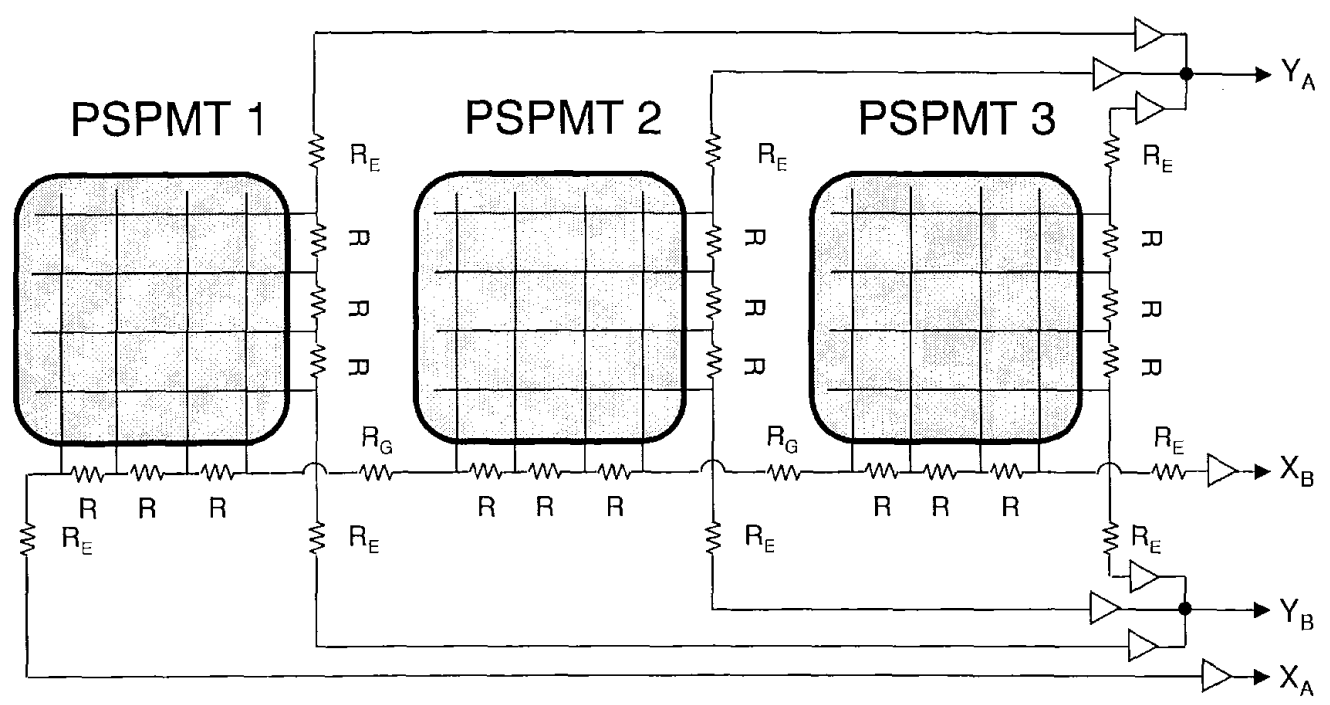

Figure 1. Scheme for combining three PSPMT detector modules through a common X-position signal divider and threc $Y$-position signal dividers. $R=100 \Omega, R_{E}=70 \Omega, R_{G}=20 \Omega$.

If these $\mathrm{X}$ and $\mathrm{Y}$ signals are used in centroid calculations to position an event, the modules will appear to form a linear array three modules (or 27 crystals) wide in the $\mathrm{X}$-direction and one module (or 9 crystals) high in the Y-direction. The two LSO crystal arrays were coupled to PSPMTs \#1 and \#3 in Figure 1 while the GSO array was coupled to PSPMT \#2. Thus, the two LSO arrays should appear at the ends of the linear array with the GSO array in the middle.

Signals from the dividers were acquired in all cases with FERA ADCs (4300B, LeCroy, Chestnut Ridge, NJ). When the detectors were studied individually, the trigger signal for the ADCs was obtained from the last dynode of each tube. When the detectors were combined, this signal was replaced by the sum of YA and YB (Figure 1) from the combined tubes. This change was necessary because of baseline drift in the dynode signal pickoff at high rates. In either case, these signals generated a valid trigger if they exceeded a low threshold set just above the system noise level. ADC offset values were acquired immediately after each experiment and subtracted from the digitized XA, XB, YA and YB signals. These corrections were necessary since small drifts in these offset values were found to significantly degrade the accuracy of event positioning particularly along the X-direction of the combined tubes (Figure 1).

Before combining the PSMPTs through the common dividers, the modules were individually field-flood illuminated at $50 \mathrm{kcps}$ with $511 \mathrm{keV}$ radiation. The modules were then combined and again field-flood illuminated with $511 \mathrm{kcV}$ radiation at 50,100, 200, 400, 600 and 800 kcps.

Images of the field-of-view of the arrays at these different rates were then analyzed to characterize (1) any inherent degradation caused by combining the tubes, and (2) the accuracy of position detection, event positioning and scintillator identification as count rate increased. The ratio of apparent crystal width to inter-crystal spacing (position detection accuracy (PDA), [5]) for the central crystal in each array was determined at the lowest count rate in each experiment and compared across the experiments. Valley-topeak ratios were determined at $50 \mathrm{kcps}$ for the central crystal in each array before the modules were combined and at 50 and $800 \mathrm{kcps}$ after the modules were combined. Valley-to-peak ratios were also determined in the gaps between detectors 1 and 2 and 2 and 3 as count rate increased. This ratio should increase with increasing pulse pileup since real events cannot occur between detector modules. Events located in the gaps must be duc to simultaneous events occurring throughout the arrays. Event mis-positioning as a function of count rate was also evaluated as count rate increased by measuring the fraction of events lost from a small spatial region-of-interest placed around an edge LSO crystal location.

Finally, the delayed charge integration method [4] was applied to separate the image data into LSO and GSO images. The DCI method is a charge integration technique in which a current signal proportional to scintillator light intensity for each event (here, the sum of YA and YB) is fully integrated and compared to a delayed integration of the same signal. It is not difficult to show that for exponentially falling signals, the full integral is related to the delayed integral by the equation:

$$
I_{F}=I_{D} \exp (T / \tau)
$$

where $\tau$ is the light decay time of the scintillator, $\mathrm{T}$ is the delay interval and $I_{F}$ and $I_{D}$ are the values of the full and delayed integrals, respectively. Equation (1) also assumes that the full integration interval is much longer than the light decay time of either scintillator.

According to (1), if the delay interval $T$ is fixed, the integrals define a straight line whose slope depends only on the light decay time of the scintillator and is independent of absorbed photon energy. Thus, if $I_{F}$ is plotted against $I_{D}$ (a "phoswich" diagram) events occurring in the same scintillator should lie on the same straight line, with low energy events near the origin and high energy events farther from the origin (since $I_{F}$ is a measure of the energy deposited in the 
scintillator). Events occurring in another scintillator with a different light decay time should also lie on a straight line radiating from the origin but with a different slope. Two such lines are, in fact, evident in Figure $2 \mathrm{~A}$ when this method is applied to data acquired in these experiments: the line with the steeper slope belongs to "fast" LSO events while the line with the shallower slope belongs to "slow" GSO events. Since events occurring in different scintillators fall on different lines or "spokes" in the phoswich diagram, regions-of-interest can be defined in the $I_{F}, I_{D}$ plane that contain events from only one kind of scintillator. If an event gives rise to a point lying in one of these defined regions, the event is assigned to the scintillator that belongs to that region. The LSO and GSO ROIs used in this study are shown in Figure 2B. These regions were drawn large simply to separate LSO from GSO events and include all but the very lowest energy events in each scintillator. The delay interval, $\mathrm{T}$, used in all experiments was $130 \mathrm{~ns}$.

The accuracy of the DCI method was assessed by first determining the total number of events falling within the LSO and GSO ROIs and then determining the fraction of these events correctly assigned to the LSO and GSO images. The correct image is known because the LSO and GSO modules are spatially distinct from one another.

\section{RESULTS}

Field-flood images $(511 \mathrm{keV}$ at $50 \mathrm{kcps})$ of the three modules coupled to their own individual resistive signal dividers and operated independently are shown in Figure $3 \mathrm{~A}$. A field-flood image of the same three modules combined as in Figure 1, also at $50 \mathrm{kcps}$, is shown in Figure 3B.
A

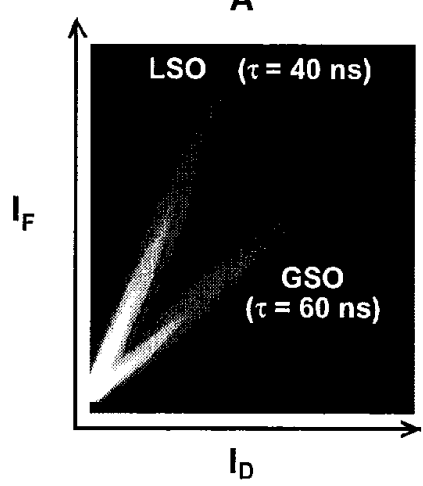

B

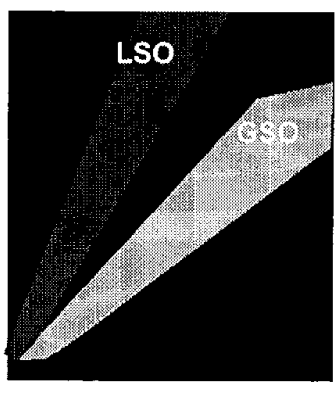

Figure 2. "Phoswich diagram" obtained by delayed charge integration (A) and ROIs used to identify the scintillator-of-interaction (B).

Position detection accuracy and valley-to-peak ratios between detectors are listed in Tables 1 and 2 for increasing count rate. The fraction of events mis-positioned due to pulse pileup as count rate increases in the combined modules is plotted in Figure 4. These measurements were made with three different ADC full-charge integration windows, 200, 300 and $600 \mathrm{~ns}$. Although the $200 \mathrm{~ns}$ results indicate improved pulse pileup rejection, spatial positioning artifacts appeared in the GSO portion of the images and these data were not considered further. Results obtained in three geometrically identical BGO modules combined in the same way are also included for comparison in Figure 4.

\section{LSO GSO LSO}

A
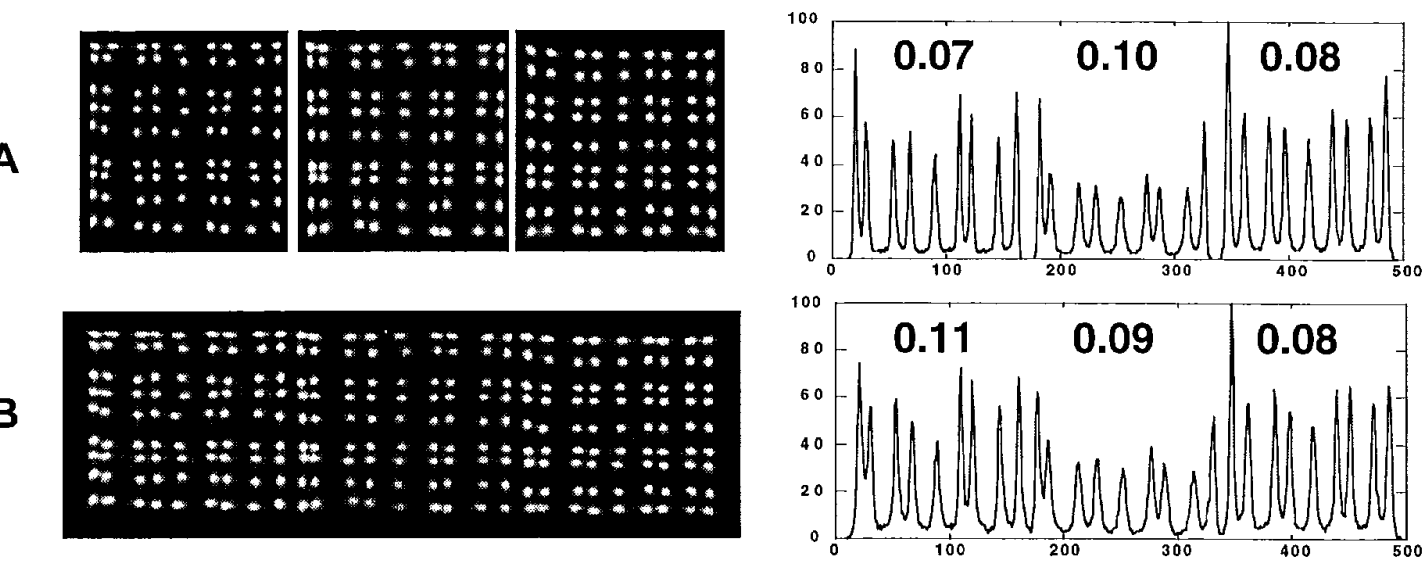

Figure 3. Field flood images acquired at $50 \mathrm{kcps}$ from each of three PSPMT modules individually (A) and when combined, (B), The corresponding count profiles across the same central row of crystals are shown at the right. Included numerical data are the valley-to-peak ratios of the central crystal in each array. 


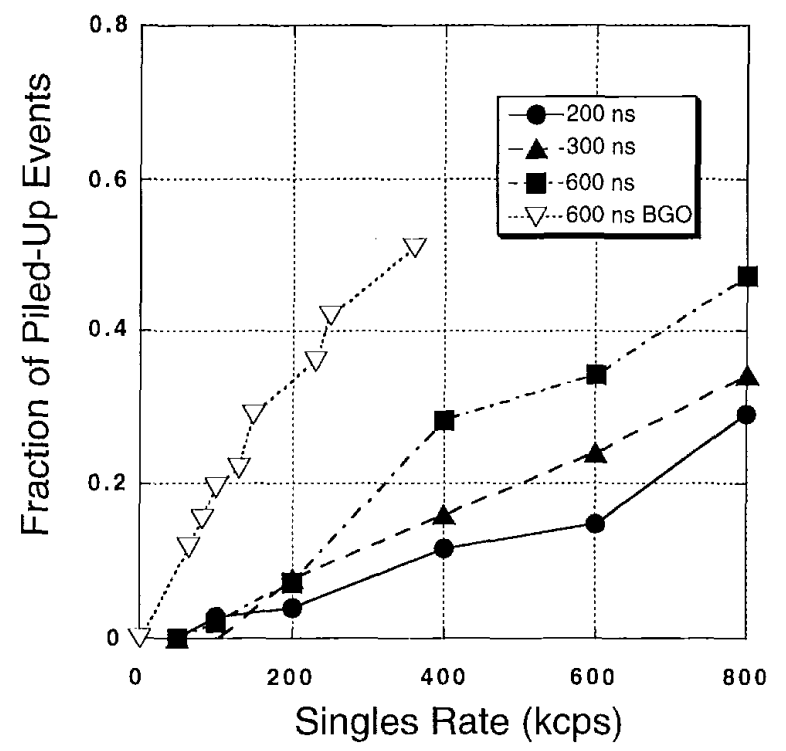

Figure 4. Fraction of events that are mis-positioned due to pulse pileup versus singles count rate in the combined modules.

The results of applying the DCI method to image data acquired from the combined LSO and GSO modules at 50 $\mathrm{kcps}$ and $800 \mathrm{kcps}$ using a $300 \mathrm{~ns}$ integration window are shown in Figures 5 and 6, respectively.

In Figure 7 the sum of the DCI-separated LSO and GSO images shown in Figure 6 is compared to the original combined image at $800 \mathrm{kcps}$. The sum of the DCI-separated images does not contain events rejected by the DCI method.
The combined image without DCI, on the other hand, contains these events.

Table 1

Position Detection Accuracy" (FWHM) for central crystal

\begin{tabular}{cccc}
$\begin{array}{c}\text { Count Rate } \\
(\mathrm{kcps})\end{array}$ & $\begin{array}{c}\text { Det. 1 } \\
\text { LSO }\end{array}$ & $\begin{array}{c}\text { Det. 2 } \\
\text { GSO }\end{array}$ & $\begin{array}{l}\text { Det. 3 } \\
\text { LSO }\end{array}$ \\
\hline $50^{\mathrm{b}}$ & 0.20 & 0.27 & 0.25 \\
50 & 0.24 & 0.27 & 0.26 \\
200 & 0.26 & 0.27 & 0.24 \\
800 & 0.32 & 0.29 & 0.25 \\
\hline
\end{tabular}

a expressed as fraction of the inter-crystal spacing $(2.2-\mathrm{mm})$ betectors illuminated individually

Table 2

Valley-to-Peak Ratios in central row profile

\begin{tabular}{ccc}
$\begin{array}{c}\text { Count Rate } \\
(\mathrm{kcps})\end{array}$ & $\begin{array}{c}\text { Ratio } \\
\text { Det. } 1 \text { and } 2\end{array}$ & $\begin{array}{c}\text { Ratio } \\
\text { Det. } 2 \text { and } 3\end{array}$ \\
\hline 50 & 0.03 & 0.05 \\
100 & 0.03 & 0.05 \\
200 & 0.05 & 0.07 \\
400 & 0.09 & 0.11 \\
600 & 0.14 & 0.15 \\
800 & 0.19 & 0.27 \\
\end{tabular}

A
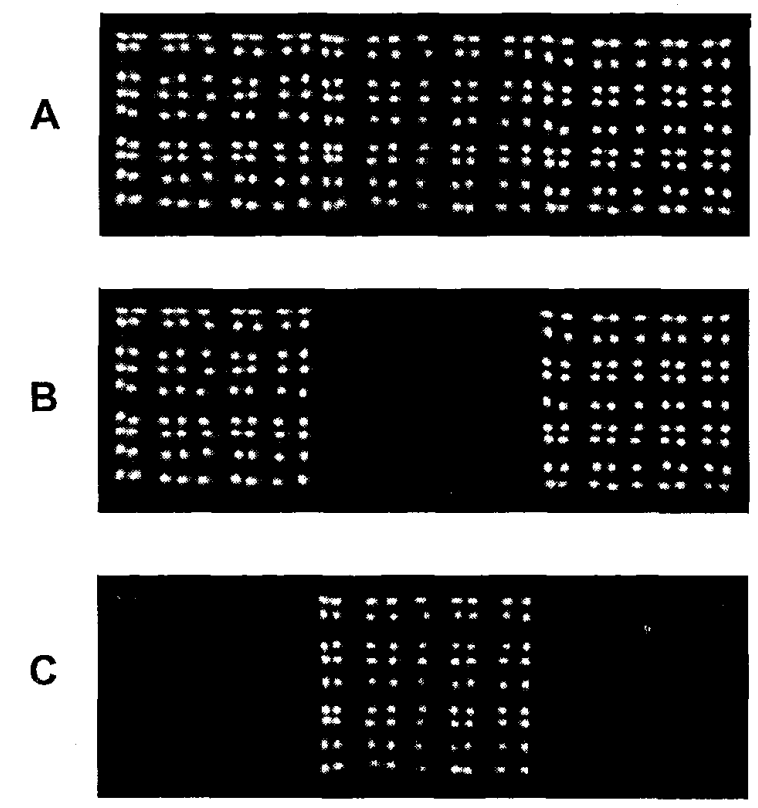

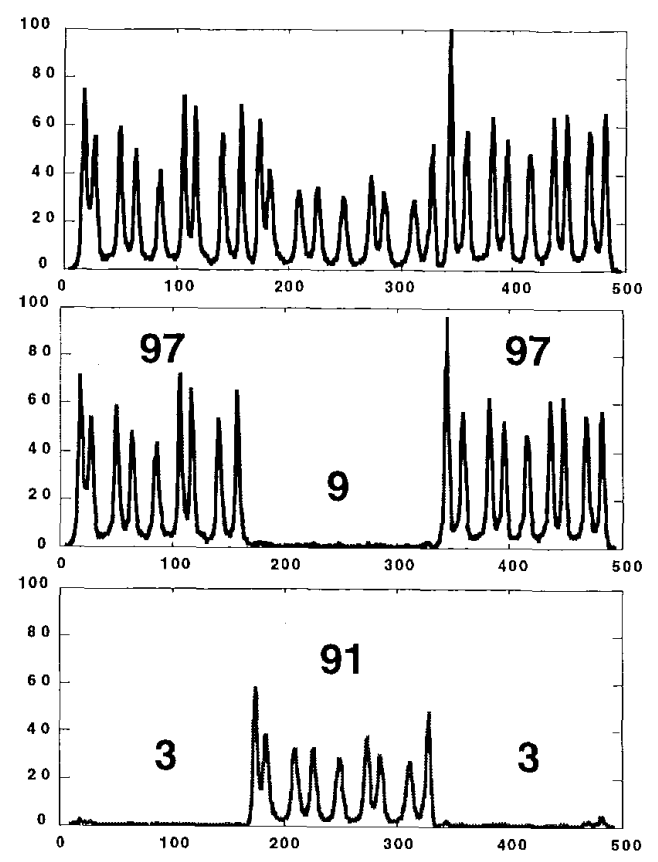

Figure 5. $50 \mathrm{kcps}, 511 \mathrm{keV}$ field-flood image of combined modules (A) using all events, (B) using events falling in the DCI LSO ROI and (C) using events falling within the DCI GSO ROI. A profile across the central row of each array is shown at the right. Included numerical data are the percentages of events assigned to each array. 

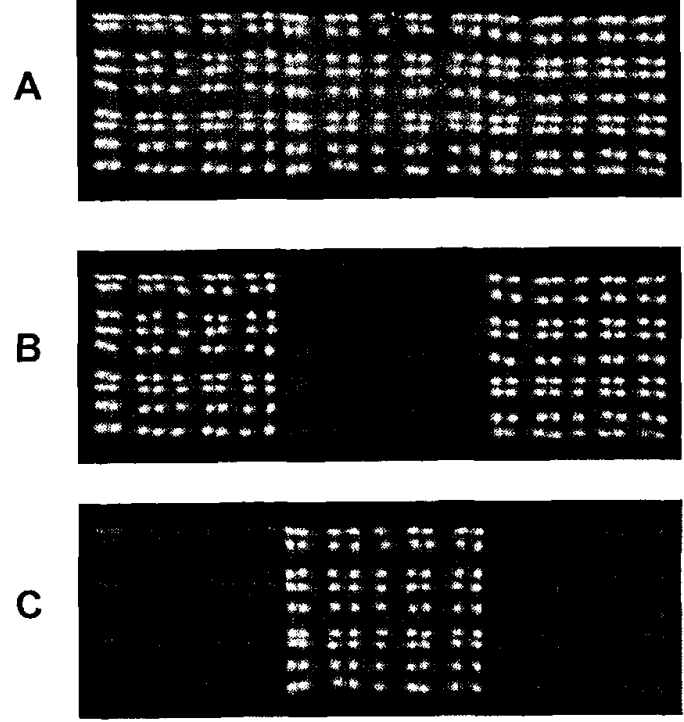
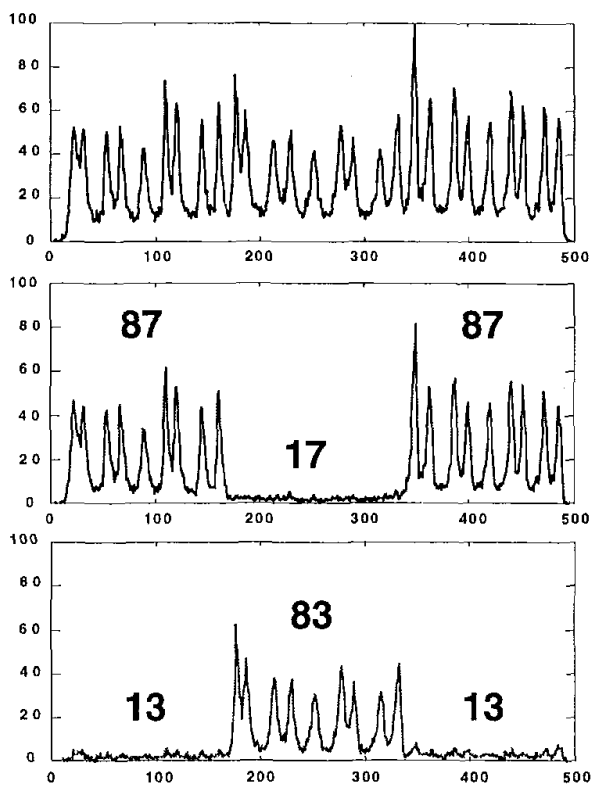

Figure 6. $800 \mathrm{kcps}, 511 \mathrm{keV}$ field-flood image before (A) and after, (B) and (C), applying the DCI method to create an LSO and GSO image, respectively. Included numerical data are the percentages of events assigned to each array.

The accuracy of the DCI method in separating LSO and GSO events at moderate and high rates is listed in Figures 5 and 6 , respectively. The fraction of GSO events incorrectly assigned to the LSO image at $50 \mathrm{kcps}$ (Figure 5B) is $9.2 \%$ of the total GSO events falling in the GSO DCI ROI. Conversely, the fraction of LSO ROI events incorrectly assigned to the GSO image (Figure 5C) after DCI is $3.1 \%$. At $800 \mathrm{kcps}$ these fractions rise to $17.3 \%$ (incorrect GSO assignments) and $13.3 \%$ (incorrect LSO assignments).

\section{DISCUSSION}

The results portrayed in Tables 1 and 2 and in Figure 3 suggest that a significant reduction in the number of signal channels can be achieved with little performance loss by combining PSPMTs through common $\mathrm{X}$ and individual $\mathrm{Y}$ resistive dividers. At $50 \mathrm{kcps}$, position detection accuracy and the valley-to-peak ratios of the central crystal in each array differ only slightly between individual and combined tubes. A careful comparison of the profiles across closely spaced peaks in Figures $3 \mathrm{~A}$ and $3 \mathrm{~B}$, however, suggests that some "fillingin" of the valleys between these peaks does occur when the tubes are combined. This result is not unexpected since PSPMT "noise" in the divider outputs of the combined tubes will be greater than for the tubes individually. While this effect is detectable, it is also small for this particular combination of PSPMTs.
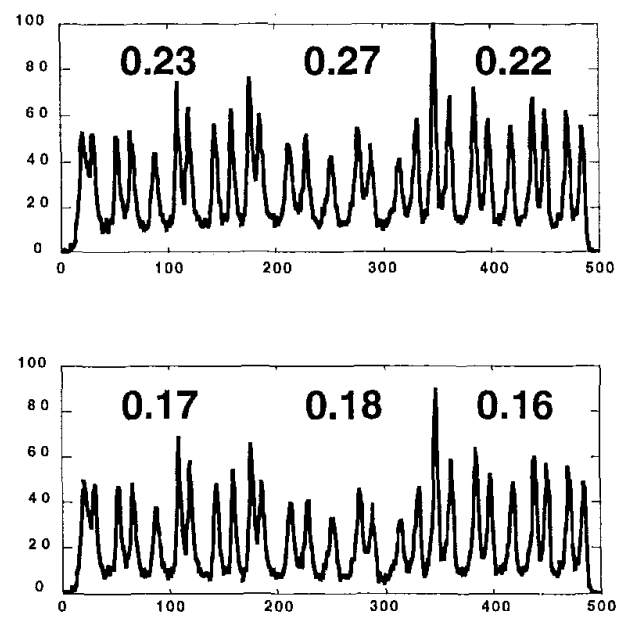

Figure 7. (A) Field-flood image at $800 \mathrm{kcps}$ shown in Figure 6A compared to the sum (B) of the DCl-separated LSO and GSO images shown in Figures $6 \mathrm{~B}$ and $6 \mathrm{C}$. Included numerical data are the valley-to-peak ratios of the central crystal in each array. 
As count rate increases, the valley-to-peak ratios in the gaps between combined detectors (Table 2) and the fraction of events mis-assigned to a crystal (Figure 4) increases. However, both of these parameters (and PDA) are degraded on average by less than $10 \%$ if total event rates are held below about $300 \mathrm{kcps}$. At higher rates ( $800 \mathrm{kcps}$, Figure 6A), pulse pileup becomes increasingly important and an increasing fraction of events are mis-positioned throughout the combined field-of-view.

The profiles shown in Figures 5 and 6 suggest that the DCI method is effective in identifying the scintillator-of-interaction over a reasonably wide range of event rates. The fraction of LSO and GSO events correctly assigned to their respective images is greater than $90 \%$ at moderate event rates and greater than $80 \%$ for count rates less than $800 \mathrm{kcps}$.

The DCI method also appears to reduce the effect of pulse pileup at high rates. The LSO and GSO sum image created after applying the DCI method (Figure 7B) exhibits a reduction in background offset compared to the original image (Figure 7A). This reduction occurs because pulse pileup will often result in events that do not fall within the LSO and GSO ROIs defined in the phoswich diagram. Indeed, any kind of event that systematically appears outside the ROIs can be rejected. For example, inter-scintillator scatter events [6] in dual-layer (LSO/GSO) phoswich modules can be rejected [4, 71 since they will contain a mixture of LSO and GSO light decay times that cause the event to be positioned between the LSO and GSO spokes. Thus, the DCI method provides an efficient one-step process for simultaneously identifying the scintillator-of-interaction while reducing the number of pileup and certain other kinds of events.

If the DCI method is implemented for each crystal in an array, rather than for the whole module (as was done here), energy windowing of LSO and GSO events can be incorporated into the analysis. The R5900-C8 PSPMT module possesses a pronounced spatial variation in gain [5]. As a result, superposition of energy spectra from throughout the field-of-view "blurs" the composite spectrum and no photopeak is obvious in either spoke of the whole-module phoswich diagram (Figure $2 \mathrm{~A}$ ). Such gain variations are negligible, however, over a region the size of a crystal and photopeaks are readily visible in the LSO and GSO spokes of phoswich diagrams for individual crystals. Unlike the ROIs shown in Figure 2B, these individual crystal ROIs would be drawn such that they covered only the photopeak region of each spoke and excluded low energy events occurring in both scintillators. The full DCI method would then be implemented as a two-step process. First, the crystal-of-interaction would be identified and the phoswich ROIs associated with that crystal retrieved. In the second step, the point defined by the full/delayed integrals for the event would be compared to these previously defined, energy-sensitive ROIs. If within either ROI, the event would be assigned to the appropriate scintillator. This two-step process results in energy windowing, scintillator identification and the rejection of pileup (and other) events. Energy windowing would also be expected to increase the accuracy of scintillator identification by removing low energy events near the origin of the phoswich diagram. Such events are likely to appear in the wrong ROI.

\section{CONCLUSIONS}

Combining up to three PSPMT detector modules through common $\mathrm{X}$ and $\mathrm{Y}$ resistive charge dividers may be an effective strategy for minimizing the cost and complexity of an imaging system comprised of large numbers of modules. Performance is little reduced at moderate event rates in the combined modules compared to the modules individually. Delayed charge integration appears effective in identifying the scintillator-of-interaction over a relatively wide range of count rates and in reducing the effects of pulse pileup at high rates. The full DCI method, scintillator identification, pileup rejection and energy windowing, can be implemented in a two-step lookup table procedure.

\section{ACKNOWLEDGEMENT}

The authors thank William Gunning (Jefferson Lab) for his expert assistance in manufacturing the PMT bases and preamplifiers used in these experiments.

\section{REFERENCES}

[1] H. O. Anger, "Scintillation Camera", Rev Sci Instr, vol. 29, pp. 27-33, 1958.

[2] H. Kume, S. Muramatsu, and M. Iida, "Position Sensitive Photomultiplier Tubes for Scintillation Imaging", IEEE Trans Nucl Sci, vol. 33, no. 1, pp. 359-363, 1986.

[3] S. Siegel, R. W. Silverman, Y. Shao, and S. R. Cherry, "Simple Charge Division Readouts for Imaging Scintillator Arrays Using a Multi-Channel PMT", IEEE Trans Nucl Sci, vol. 43, no. 3, pp. 1634-1641, 1996.

[4] J. Seidel, J. J. Vaquero, S. Siegel, W. R. Gandler, and M. V. Green, "Depth Identification Accuracy of a ThreeLayer Phoswich PET Detector Module", IEEE Trans Nucl Sci, vol. 46, no. 3, pp. 485-490, 1999.

[5] J. J. Vaquero, J. Seidel, S. Siegel, W. R. Gandler, and M. V. Green, "Performance Characteristics of a Compact Position-Sensitive LSO Detector Module", IEEE Trans Med Imag, vol. 17, no. 6, pp. 967-978, 1998.

[6] Y. Shao, S. R. Cherry, S. Siegel, and R. W. Silverman, "A Study of Inter-Crystal Scatter in Small Scintillator Arrays Designed for High Resolution PET Imaging", IEEE Trans Nucl Sci, vol. 43, no. 3, pp. 1938-1944, 1996.

[7] M. Schmand, L. Eriksson, M. E. Casey, K. Wienhard, G. Flügge, and R. Nutt, "Advantages Using Pulse Shape Discrimination to Assign the Depth of Interaction Information (DOI) from a Multi Layer Phoswich Detector", IEEE Trans Nucl Sci, vol. 46, no. 4, pp. 985 990, 1999. 